\title{
Temperature Dependence of a Macrobending Edge Filter Based on a High-bend Loss Fiber
}

\author{
Pengfei Wang \\ Technological University Dublin, pengfei.wang@tudublin.ie \\ Ginu Rajan \\ Technological University Dublin \\ Yuliya Semenova \\ Technological University Dublin, yuliya.semenova@tudublin.ie
}

See next page for additional authors

Follow this and additional works at: https://arrow.tudublin.ie/engscheceart

Part of the Engineering Commons

\section{Recommended Citation \\ Wang, P., Rajan, G., Semenova, Y., Farrell, G. (2008) Temperature dependence of a macrobending edge filter based on a high-bend loss fiber. Optics Letters, Vol. 33, No. 21, pp. 2470-2472, 2008. doi:10.1364/ OL.33.002470}

This Article is brought to you for free and open access by the School of Electrical and Electronic Engineering at ARROW@TU Dublin. It has been accepted for inclusion in Articles by an authorized administrator of ARROW@TU Dublin. For more information, please contact arrow.admin@tudublin.ie, aisling.coyne@tudublin.ie, gerard.connolly@tudublin.ie.

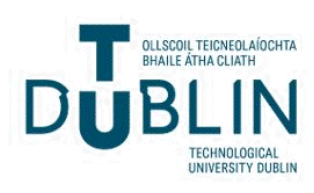


Authors

Pengfei Wang, Ginu Rajan, Yuliya Semenova, and Gerald Farrell

This article is available at ARROW@TU Dublin: https://arrow.tudublin.ie/engscheceart/50 


\title{
Temperature dependence of a macrobending edge
}

\section{filter based on a high-bend loss fiber}

\author{
Pengfei Wang,* Ginu Rajan, Yuliya Semenova, Gerald Farrell \\ Applied Optoelectronics Center, School of Electronic and Communications \\ Engineering, Dublin Institute of Technology, Kevin Street, Dublin 8, Ireland \\ Corresponding author: pengfei.wang@student.dit.ie
}

\begin{abstract}
The temperature dependence of a macrobending bare fiber based edge filter is investigated both theoretically and experimentally. The fiber used is a high bend loss fiber, type 1060XP. The experimental results show a good agreement with the proposed theoretical model over a temperature range from 0 to $80^{\circ} \mathrm{C}$. It is shown that the strong temperature dependence of a high-bend loss fiber has a significant influence on the performance of a fiber edge filter used in a wavelength measurement application. However it is also concluded that such a temperature dependent performance can be beneficially utilized in a fiber temperature sensing application.
\end{abstract}

OCIS codes $060.2310,120.2440,120.6780$

A singlemode bent fiber can provide a range of useful characteristics which can be utilized, for example, in novel fiber components such as sensors and edge filters [1-5] or used to enhance the operation of fiber lasers. Such bent fiber based components have the advantages of ease of fabrication and simplicity of interconnection to other fibers.

Recently, in an application involving an edge filter for rapid wavelength measurement, the macrobending loss of multiple turns of SMF28 fiber has been investigated theoretically and experimentally $[4,5]$ in order to optimize the structure. Such SMF28 based edge filter includes a splitter, a bending fiber based edge filter, a reference arm and two photodetectors. One splitter arm connects with the edge filter, while the other acts as a reference arm. Both arms are terminated in photodetectors to optical power levels. Given the wavelength dependence of bend loss, the ration of the measured power levels is a fraction of wavelength. Thus with a suitable calibration wavelength can be measured. However, in our previously published investigations [6, 
7], it was found that the fiber polymer coating layer, which provides mechanical protection for the fiber, has a significant influence on both fiber bending loss and polarization dependent loss. For the fiber bend loss edge filter application, such coating layer(s) can also induce random variations throughout the measured transmission spectrum [8].

To obtain the desired transmission spectrum with a shorter fiber bend length for more compact structure, a type of bending loss sensitive fiber is desired, where the normalized frequency $V$ should be lower. Furthermore, it was found that polarization dependent loss could reduce the precision of wavelength measurement. Therefore, in order to ensure accuracy in wavelength measurement, low polarization dependence for the fiber bending loss is required as well.

In terms of such requirements of optical fiber for fiber edge filter mentioned above, an optimized macrobending fiber edge filter based on a 1060XP fiber with single turn structure has been developed and presented [9]. 1060XP fiber is a high-bend loss fiber, originally developed for fiber laser applications. The fiber is stripped of its coating layer to remove the influence of the fiber coatings and has an optical absorption layer applied to the cladding. By comparison with SMF28 fiber presented previously [5], both theoretical and experimental results have shown that the 1060XP fiber based edge fiber can effectively remove the coating(s) induced influences mentioned above, and the bending loss of bare 1060XP fiber with absorbing layer can achieve a marginally better polarization dependent loss behavior than an equivalent SMF28 based edge filter, with the added benefit of a more compact physical structure (22 turns of SMF28 with radius for $11 \mathrm{~mm}$ by comparison with single turn of 1060XP with a bending radius of $10.5 \mathrm{~mm}$ for a typical edge filter). In the experiments, when a bare 1060XP fiber (bend radius is $10.5 \mathrm{~mm}$, and the fiber length is single turn) is employed for ratiometric wavelength measurement, it is found that the system's resolution is approximately 10 picometer at $1550 \mathrm{~nm}$ when the fluctuation of the ratio caused by the noise of the photodetectors is approximately $0.002 \mathrm{~dB}$.

In practice, in the experiments in [8] it was found that changes in temperature affected the measured bending loss. Upon investigation it was realized that because the bend loss of 1060XP is approximately two times more temperature sensitive than SMF28, so the spectral response of an edge filter based on 1060XP fiber would be more temperature dependent. Used in a wavelength measurement system, temperature changes could adversely affect the accuracy of the wavelength measurement system. There is a need therefore to properly quantify the temperature dependence of 1060XP fiber, to determine the extent of the effect on the spectral response of an edge filter so as to develop solutions such as active temperature control of the filter or ambient temperature feedback to adjust the calibration of the system as temperature changes. The temperature dependent properties of 1060XP bent fiber also suggest a possibility of designing and developing a novel temperature sensor based on a simple bending fiber structure, with the fiber bend loop optimized as an optical fiber probe with small bending radii.

In this letter, the temperature dependent loss (TDL) behavior for the key edge 
filter parameters, baseline loss and discrimination range, for a 1060XP based edge filter are investigated and presented. The investigation involves both theoretical modeling of TDL and experimental verification. The theoretical numerical modeling shows a good agreement with the measured results for the TDL of bent 1060XP fiber with a correction factor (namely, the wavelength dependent effective bend radius, which is employed to achieve good agreement between the modeled results and the experimental results due to the fiber bending stress) of 1.298 at a wavelength of 1550 $\mathrm{nm}$.

In previously published work [9], for a fiber bend loss edge filter application, the bare cladding fiber was coated with an absorbing layer. Analytically this is approximately equivalent to a fiber structure with an infinite cladding and the expression for calculating the fiber bend loss coefficient with an infinite cladding has been expressed by Marcuse in Ref. [10]. Furthermore, in the theoretical model, the so-called effective bend radius (correction factor) presented in previously published papers [11-13] has been utilized in the calculated model of bending loss to fit the experimental results.

For the silica material used in the fiber, the physical expansion or contraction of the material's volume in response to temperature changes is characterized by silica's thermal expansion coefficient (TEC).

The refractive index of an optical material depends on temperature and wavelength. For silica material, when the ambient temperature changes the consequent refractive index variations of the silica glass material, caused by lattice deformation of its matrix structure are characterised by the thermo-optic coefficient (TOC). Therefore, in the theoretical modelling which is based on the scalar approximation method developed by Marcuse [10], both TEC and TOC are accounted for in the calculated prediction for bending loss.

For 1060XP singlemode fiber, the corresponding parameters are shown in Table I:

Previously, the presented fiber bend loss edge filter [4, 5] utilized for a measurable wavelength range from $\lambda_{1}$ to $\lambda_{2}$, provides a strong monotonically increasing wavelength dependent attenuation range, which can be defined as discrimination range, the power difference between a given lower start wavelength $\lambda_{1}$ (at which the attenuation value is the so-called baseline loss) and an end wavelength $\lambda_{2}$. These two parameters, baseline loss and discrimination range, can be used to effectively evaluate the performance of a fiber edge filter.

To gain an insight into the influence of temperature on the baseline loss (bend loss at $1500 \mathrm{~nm}$ ) and discrimination range (the difference of bend loss between 1500 and $1600 \mathrm{~nm}$ ), the calculated baseline loss and discrimination range as a function of different bend radii at a temperature of $0^{\circ} \mathrm{C}$ and $80^{\circ} \mathrm{C}$, is presented in Fig. 1.

In relation to the choice of temperature range, it is accepted that such a wide temperature range would be very rare in a wavelength measurement system, typically used in an ambient environment. However as mentioned above, since there are potential temperature sensing applications, where wider temperature ranges could be expected, the temperature end-points throughout the investigation were chosen to 
match the maximum temperature range possible in the experimental setup available, that is $0^{\circ} \mathrm{C}$ to $80^{\circ} \mathrm{C}$.

As shown in Fig. 1, one can see that if the temperature changes from $0^{\circ} \mathrm{C}$ to $80^{\circ} \mathrm{C}$, significant changes occur in both baseline loss and discrimination range, with a consequent impact on the spectral response of a 1060XP based edge filter. For example, for a selected bend radius of $10.5 \mathrm{~mm}$ [9], the baseline loss changes from $5.785 \mathrm{~dB}$ to $4.604 \mathrm{~dB}$, and the discrimination range changes from $18.48 \mathrm{~dB}$ to 15.698 $\mathrm{dB}$ when temperature changes from $0^{\circ} \mathrm{C}$ to $80^{\circ} \mathrm{C}$.

In this work, the TDL value is defined as an absolute value of the difference in bend loss between $0^{\circ} \mathrm{C}$ and a temperature of $T^{\circ} \mathrm{C}$, where $T$ can vary from $0^{\circ} \mathrm{C}$ to $80^{\circ} \mathrm{C}$, thus:

$$
T D L=\left|B L_{T^{o} C}-B L_{0^{o} C}\right|
$$

Choose the 1060XP fiber as an edge filter example and one bending radius is considered. The TDL of bending bare 1060XP fiber with a bend radius of $10.5 \mathrm{~mm}$ at a wavelength of $1550 \mathrm{~nm}$ is calculated based on the theoretical model presented in Ref. [10]. In Fig. 2, it is shown that the modeled TDL results (Plot A) demonstrate a strong monotonic increase from a start temperature $\left(0^{\circ} \mathrm{C}\right)$ to an end temperature $\left(80^{\circ} \mathrm{C}\right)$. For a SMF28 fiber base edge filter [8], it is found that the TDL display several strong periodical bending loss response at different bend radii due to whispering gallery modes (WGMs) effects guided at cladding-coating interface, whereas for bare 1060XP fiber presented in Fig. 2, it is clearly that the TDL changes monotonically over the measured temperature range from a start temperature $\left(0^{\circ} \mathrm{C}\right)$ to an end temperature $\left(80^{\circ} \mathrm{C}\right)$, such TDL performance is significantly different with that of coated SMF28, this indicates that temperature variations induced periodical bending loss response of coated fiber is mainly caused by coatings, and the striped bare fiber with absorbing layer has effectively reduced the impact of WGMs.

In order to verify the theoretical model for TDL, an experimental setup for measuring TDL was developed and is shown in Fig. 3. The bending fiber was connected to a tunable laser and an optical spectrum analyzer. The tunable laser has an output power of $0 \mathrm{dBm}$. The bare 1060XP fiber section coated with an absorbing layer (acrylic based material) is bent to form a small $360^{\circ}$ bend, and is mounted on a thermoelectric Peltier cooler using a two-part epoxy to improve mechanical stability. The measured bend radius is $10.5 \mathrm{~mm}$. The Peltier cooler is controlled by a digital temperature controller (ITC 510, Thorlabs), and a digital platinum resistance thermometer sensor probe is attached to the Peltier cooler to accurately measure the temperature.

The TDL of the 1060XP fiber was measured for a bending radius of $10.5 \mathrm{~mm}$ at a wavelength of $1550 \mathrm{~nm}$. The experimental TDL results of 1060XP as a function of the temperature range from 0 to $80^{\circ} \mathrm{C}$ are presented in Fig. 2 (Plot B).

In Fig. 2, one can also see that there is a good agreement between the calculated (Plot A) and experimental data (Plot B). The perceptible divergence between the experimental and theoretical results may be caused by: 1) the limitations of the absorbing layer on the surface of the bare fiber where the absorbing layer does not 
absorb all the radiation lost at the bend at the fiber cladding-air interface, leading to WGMs effects, such WGMs can recouple to the propagating mode in the fiber core and can result in wavelength dependent interference effects, further results in the perceptible quasi-periodic behavior of the measured results in Fig. 2. Further investigation using different absorbing layer materials is ongoing; 2) minute discrepancies between the temperature of the fiber and the reference thermometer sensor probe, for example those resulting from ambient airflow.

To better illustrate and evaluate the temperature influence of a macrobending-based 1060XP fiber edge filter as a wavelength measurement application over the whole wavelength range from 1500 to $1600 \mathrm{~nm}$, the theoretical and experimental macrobending loss differences between $0^{\circ} \mathrm{C}$ and $80^{\circ} \mathrm{C}$ over the wavelength range from 1500 to $1600 \mathrm{~nm}$ for a designed bend radius of $10.5 \mathrm{~mm}$ are presented in Fig. 4. The correction factors determined at $10 \mathrm{~nm}$ intervals over the wavelength range of interest between 1500 and $1600 \mathrm{~nm}$ are used in the TDL theoretical model. As shown in Fig. 4, one can see that there is very good match between the calculated and experimental data. The experimental TDL data shows a monotonically increasing characteristic which matches the proposed theoretical predictions well. The measured TDL is about $1.19 \mathrm{~dB} /$ turn at a wavelength of 1500 $\mathrm{nm}$ and is circa $4.02 \mathrm{~dB} /$ turn at a wavelength of $1600 \mathrm{~nm}$.

In conclusion, based on a scalar approximation theory, we have studied and investigated the temperature dependence of bare macrobending 1060XP fiber theoretically and experimentally, for an all-fiber bending loss edge filter application. The experimental results show a good agreement with the proposed theoretical model. For 1060XP fiber based edge filter, it is clear that temperature variations will significantly impact the accuracy of wavelength measurements. Our method can be used to evaluate the performance of a fiber macrobending based edge filter as a function of ambient temperature for high-speed wavelength measurements and also for fiber-based temperature sensing applications.

\section{Reference}

1. R. Morgan, J. S. Barton, P. G. Harper and J. D. C. Jones, "Temperature dependence of bending loss in monomode optical fibres," Electron. Lett. 26(13), 937-939 (1990).

2. F. M. Haran, J. S. Barton, S. R. Kidd and J. D. C. Jones, "Optical fibre interferometric sensors using buffer guided light," Meas. Sci. Technol. 5(5), 526-530 (1994).

3. S. H. Nam, and S. Yin, "High-Temperature Sensing Using Whispering Gallery Mode Resonance in Bent Optical Fibers," IEEE Photon. Technol. Lett., 17, 2391-2393 (2005).

4. Q. Wang, G. Farrell and T. Freir, "Study of transmission response of edge filters employed in wavelength measurements," Appl. Opt., 44(36), 7789-7792 (2005).

5. Q. Wang, G. Farrell, T. Freir, G. Rajan, and P. Wang, "Low-cost wavelength measurement based on a macrobending single-mode fiber," Opt. Lett., 31(12), 
1785-1787 (2006).

6. Q. Wang, G. Farrell, and T. Freir, "Theoretical and experimental investigations of macro-bend losses for standard single mode fibers," Opt. Express, 13(12), 4476-4484 (2005).

7. Q. Wang, G. Rajan, P. Wang, and G. Farrell, "Polarization dependence of bend loss for a standard singlemode fiber," Opt. Express 15(8), 4909-4920 (2007).

8. P. Wang, Y. Semenova, and G. Farrell, "Temperature dependence of macrobending loss in all-fiber bend loss edge filter," Opt. Commun., 281, 4312-4316 (2008).

9. P. Wang, G. Farrell, Q. Wang, G. Rajan, “An optimized macrobending-fiber-based edge filter," IEEE Photon. Technol. Lett., 19(15), 1136-1138 (2007).

10. D. Marcuse, "Curvature loss formula for optical fibers," J. Opt. Soc. Am. 66(3), 216-220 (1976).

11. A. B. Sharma, A. -H. Al-Ani and S. J. Halme, "Constant-curvature loss in monomode fibers: an experimental investigation," Appl. Opt. 23(19), 3297-3301, (1984).

12. H. Renner, "Bending losses of coated single-mode fibers: a simple approach," J. Lightw. Technol., 10(5), 544-551, (1992).

13. L. Faustini and G. Martini, "Bend loss in single-mode fibers," J. Lightw. Technol., 15(4), 671-679, (1997).

\section{Figure captions}

Fig. 1 Calculated baseline loss and discrimination range at $0^{\circ} \mathrm{C}$ and $80^{\circ} \mathrm{C}$, for a fiber 
length of one turn, with correction factors of $1.308 @ 1500 \mathrm{~nm}$ and 1.336@1600 nm.

Fig. 2 Calculated (Plot A) and measured (Plot B) TDL for a temperature range from 0 to $80^{\circ} \mathrm{C}$ at a wavelength of $1550 \mathrm{~nm}$ with a bending radius of $10.5 \mathrm{~mm}$ and a single fiber turn.

Fig. 3 Experimental setup for measuring the TDL of a bent 1060XP fiber.

Fig. 4 Calculated and measured temperature dependence between 0 and $80^{\circ} \mathrm{C}$ as a function of wavelength (with an interval of $10 \mathrm{~nm}$ ), for an edge filter with a bend radius of $10.5 \mathrm{~mm}$ and a single turn of fiber.

\section{Table Caption}

TABLE I: PARAMETERS OF THE 1060XP FIBER; 



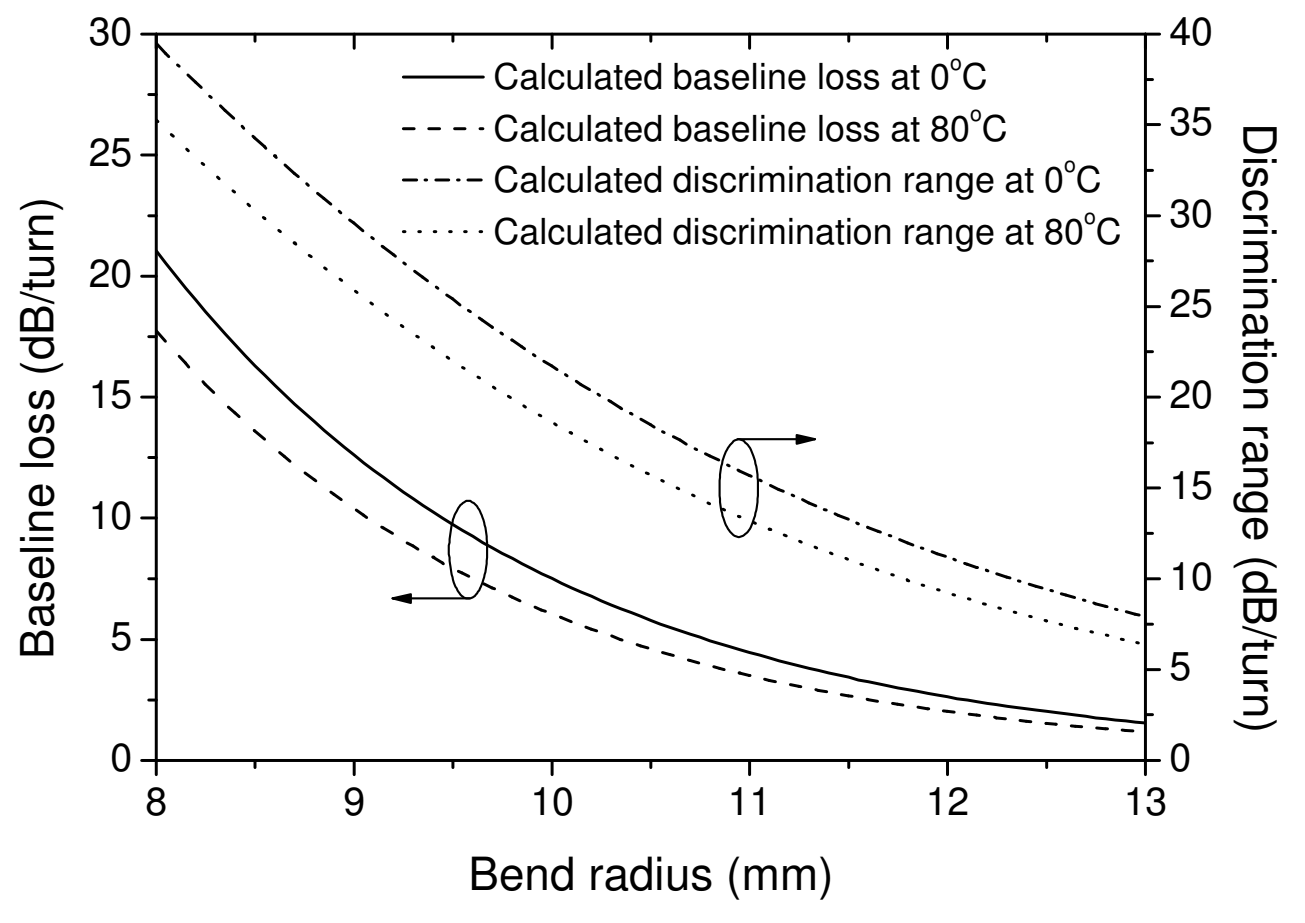

Fig. 1 


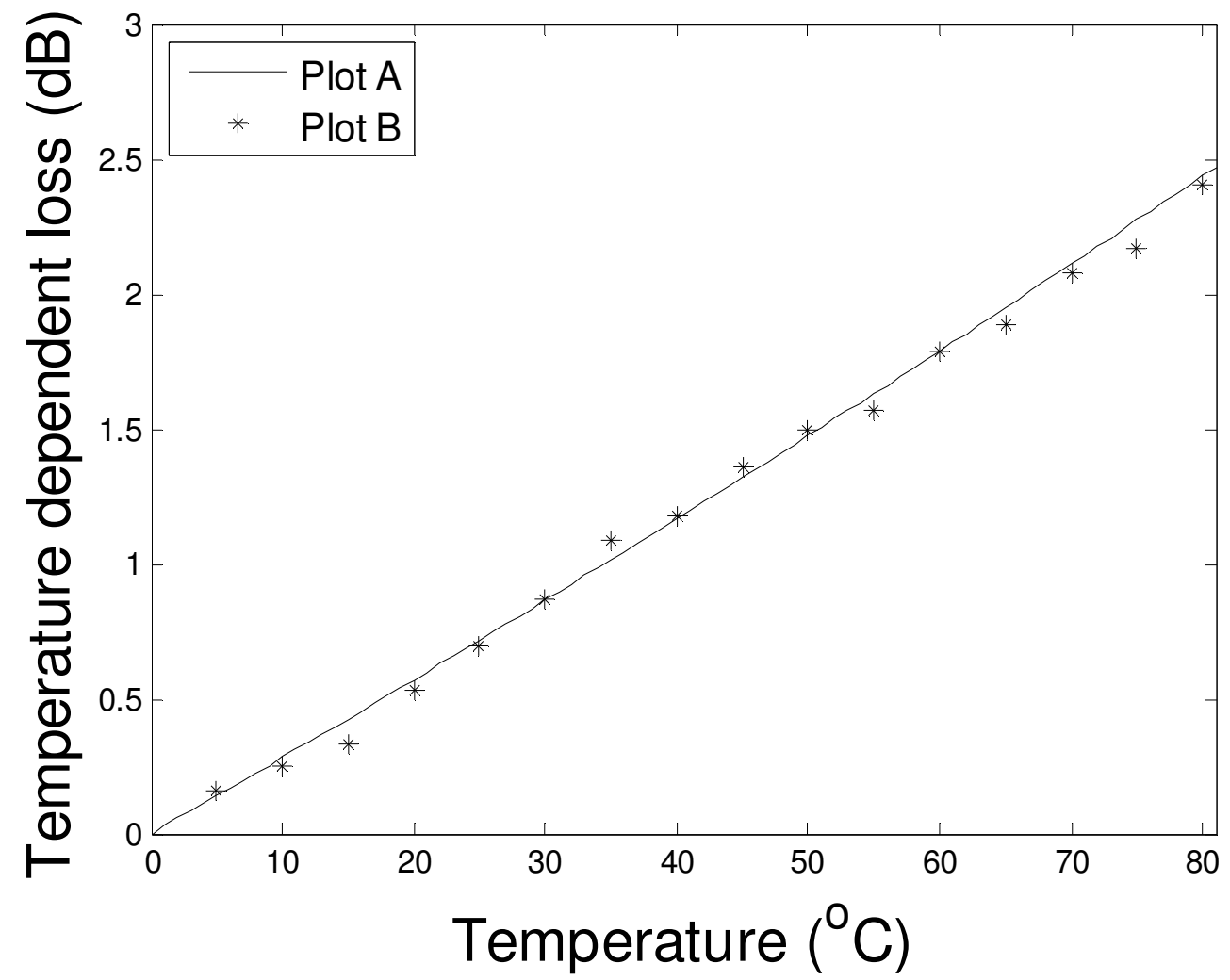

Fig. 2 


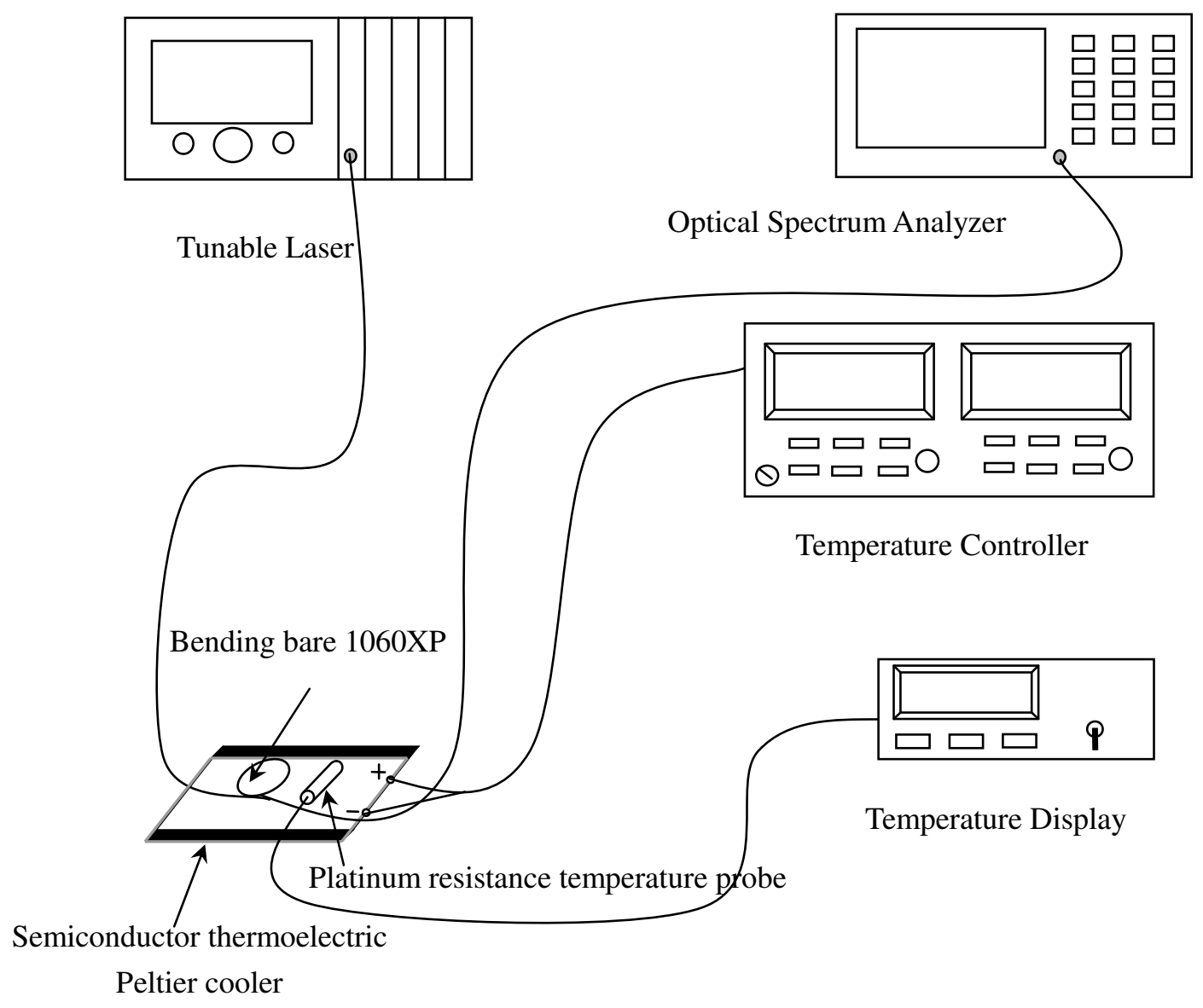

Fig. 3 


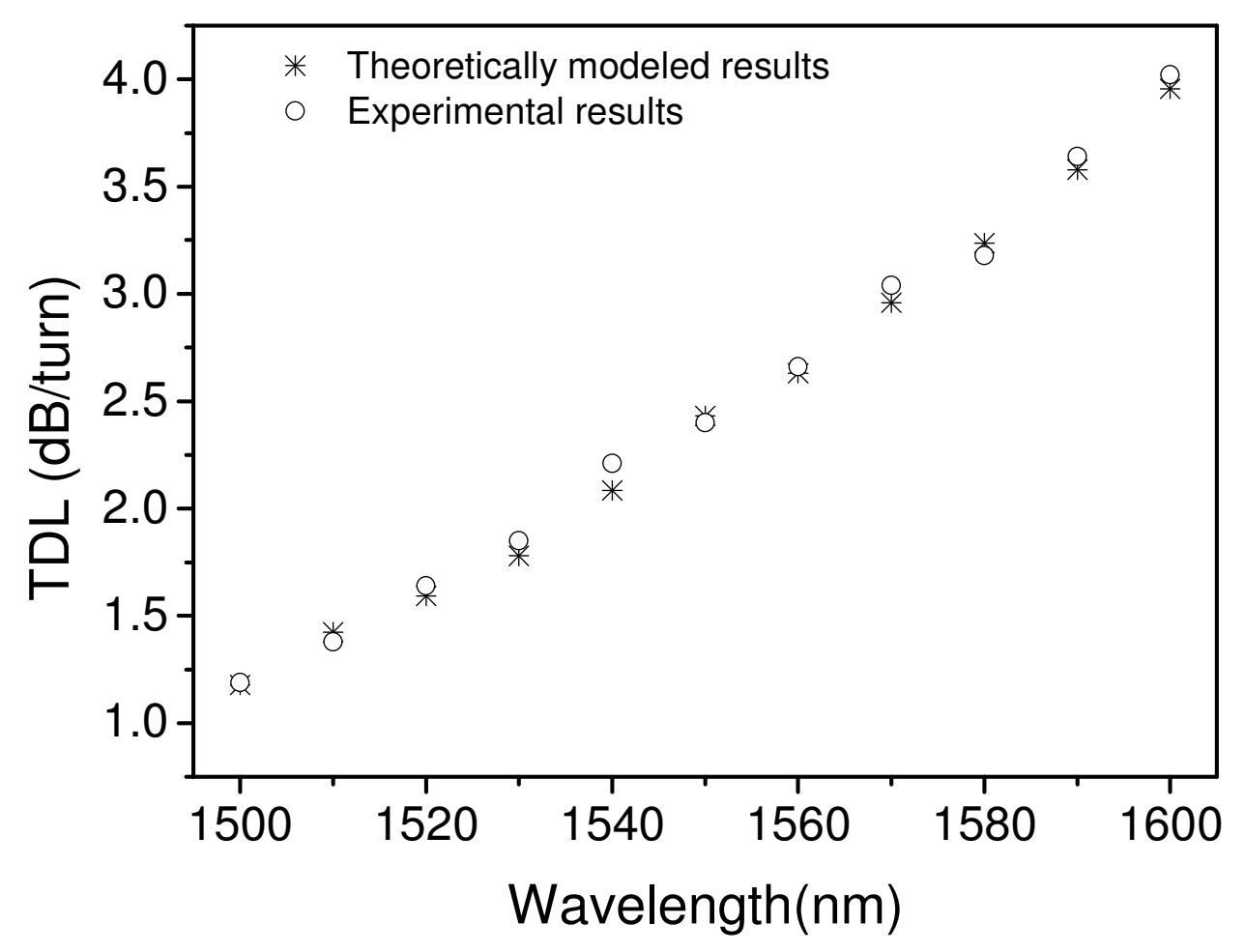

Fig. 4 
TABLE I: PARAMETERS OF THE 1060XP FIBER;

\begin{tabular}{ccc}
\hline \hline 1060XP fiber & Core & Cladding \\
\hline Diameter $(\mu \mathrm{m})$ & $5.3 \pm 0.5$ & $125 \pm 0.5$ \\
TEC $\alpha\left(\mathrm{K}^{-1}\right)$ & $\sim 5.5 \times 10^{-7}$ & - \\
$\operatorname{TOC} \beta\left(\mathrm{K}^{-1}\right)$ & $\sim 1.1 \times 10^{-5}$ & $\sim 1 \times 10^{-5}$ \\
\hline \hline
\end{tabular}

